\title{
EXACT SOLUTIONS OF A FAMILY OF HIGHER-DIMENSIONAL SPACE-TIME FRACTIONAL KDV-TYPE EQUATIONS
}

\author{
MOHAMMED O. AL-AMR \\ Department of Mathematics, College of Computer Sciences and Mathematics, \\ University of Mosul, Mosul 41002, Iraq
}

\begin{abstract}
In this paper, based on the definition of conformable fractional derivative, the functional variable method (FVM) is proposed to seek the exact traveling wave solutions of two higherdimensional space-time fractional KdV-type equations in mathematical physics, namely the $(3+1)$-dimensional space-time fractional Zakharov-Kuznetsov $(Z K)$ equation and the $(2+1)$ dimensional space-time fractional Generalized Zakharov-Kuznetsov-Benjamin-Bona-Mahony $(G Z K-B B M)$ equation. Some new solutions are procured and depicted. These solutions, which contain kink-shaped, singular kink, bell-shaped soliton, singular soliton and periodic wave solutions, have many potential applications in mathematical physics and engineering. The simplicity and reliability of the proposed method is verified.
\end{abstract}

\section{KEYWORDS}

Functional Variable Method, Fractional Partial Differential Equations, Exact Solutions, Conformable Fractional Derivative

\section{INTRODUCTION}

In recent years, Fractional partial differential equations (FPDEs) have been extensively utilized to model complex physical phenomena that arise in various aspects of science and engineering, such as applied mathematics, physics, chemistry, biology, signal processing, control theory, finance and fractional dynamics [1,2]. The analytical solutions of FPDEs play a significant role in the study of nonlinear physical phenomena. Therefore, the efficient approaches to construct the solutions of FPDEs have attracted great interest by several groups of researchers. A large collection of analytical and computational methods has been introduced for this reason, for example the exp-function method [3,4], Adomian decomposition method [5], the $\left(G^{\prime} / G\right)$ expansion method [6], the first integral method [7,8], the variational iteration method [9], the subequation method [10,11], the modified simple equation method [12], Jacobi elliptic function expansion method [13], the generalized Kudryashov method [14,15] and so on. One of the most powerful methods for seeking analytical solutions of nonlinear differential equations is the functional variable method, which was first proposed by Zerarka et al. [16,17] in 2010. It has received much interest since it has been employed to solve a wide class of problems by many authors [18-22]. The main advantage of this method over other existing methods is its capability 
to reduce the size of computations during the solution procedure. Therefore, it can be applied without using any symbolic computation software.

As one of the most well-known nonlinear dispersive equations, the Korteweg-de Vries (KdV) equation has attracted much attention by many researchers in the scientific community duo to its significant role in various scientific disciplines. It describes a variety of important nonlinear phenomena, including finite amplitude dispersive wave phenomena, acoustic waves in a harmonic crystal and ion-acoustic waves in plasmas [23]. Several variations of this equation have been introduced in the literature. The (3+1)-dimensional Zakharov-Kuznetsov (ZK) equation was derived as a three-dimensional generalization of the $\mathrm{KdV}$ equation, which arises as a model for the propagation of nonlinear plasma-acoustic waves in the isothermal multi-component magnetized plasma [24,25]. If the nonlinear dispersion in $\mathrm{KdV}$ equation is incorporated, the Benjamin-Bona-Mahony (BBM) equation arises to describe a propagation of long waves. The (2+1)-dimensional Generalized Zakharov-Kuznetsov-Benjamin-Bona-Mahony (GZK-BBM) equation was developed by Wazwaz [26] as a combination of the well-known Benjamin-BonaMahony (BBM) equation with the ZK equation. It arises as a description of gravity water waves in the long-wave regime. Therefore, it is very interesting to examine the traveling wave solutions of KdV-type equations. It is worthwhile to mention that the $(3+1)$-dimensional space-time fractional ZK equation and the (2+1)-dimensional space-time fractional GZK-BBM equation have not been solved yet by using any existing analytical method.

There are many different definitions for fractional differential equations in fractional calculus; among these definitions are Riemann-Liouville, Grünwald-Letnikov, Caputo, Weyl, Marchaud, Hadamard, Canavati, Davidson-Essex, Riesz-Fischer, Jumarie fractional derivatives and so on [2,27]. However, these definitions have some shortcomings. For instance, they do not satisfy the product rule, the quotient rule and the chain rule for derivative operations. To overcome these drawbacks, Khalil et al. [28] introduced a completely new definition of the fractional derivative, which is more natural and fruitful than previous ones, called conformable fractional derivative.

The present paper is devoted to suggest the functional variable method for constructing new exact solutions of two higher-dimensional space-time fractional $\mathrm{KdV}$-related equations, namely the $(3+1)$-dimensional space-time fractional $\mathrm{ZK}$ equation and the $(2+1)$-dimensional space-time fractional GZK-BBM equation. The fractional derivatives are presented in terms of the conformable sense. To the best of our knowledge, these equations have not been investigated previously by using the functional variable method in the sense of conformable derivative.

The rest of the paper is organized as follows: In Section 2, we describe some relevant materials and methods. In Section 3, the proposed approach is applied to establish the exact solutions of the underlying equations. The graphical representations of the obtained solutions are provided in Section 4, Results and discussion are presented in Section 5. Finally, conclusions are given in Section 6.

\section{MATERIALS AND METHODS}

\subsection{Conformable fractional derivative and its properties}

In this subsection, we present some basic definitions and properties of the conformable fractional calculus. Suppose a function $f:[0, \infty) \rightarrow \square$, then, the conformable fractional derivative of order $\alpha$ is defined as follows [28,29]: 
$T_{\alpha}(f)(t)=\lim _{\varepsilon \rightarrow 0} \frac{f\left(t+\varepsilon t^{1-\alpha}\right)-f(t)}{\varepsilon}$,

in which $t>0$ and $0<\alpha \leq 1$. If $f$ is $\alpha$-differentiable in some $(0, a), a>0$, and $\lim _{t \rightarrow 0^{+}} f^{(\alpha)}(t)$ exists, then $f^{(\alpha)}(0)=\lim _{t \rightarrow 0^{+}} f^{(\alpha)}(t)$.

Now, we summarize some useful properties of the conformable derivative as follows [28-30]:

(i) $\quad T_{\alpha}(a f+b g)=a T_{\alpha}(f)+b T_{\alpha}(g)$, for all $a, b \in \square$.

(ii) $T_{\alpha}\left(t^{p}\right)=p t^{p-\alpha}$, for all $p \in \square$.

(iii) $\quad T_{\alpha}(f g)=f T_{\alpha}(g)+g T_{\alpha}(f)$.

(iv) $T_{\alpha}\left(\frac{f}{g}\right)=\frac{g T_{\alpha}(f)-f T_{\alpha}(g)}{g^{2}}$.

(v) $T_{\alpha}(\lambda)=0$, where $\lambda$ is a constant.

(vi) If $f$ is differentiable, then $T_{\alpha}(f)(t)=t^{1-\alpha} \frac{d f}{d t}$.

(vii) If $f, g$ are differential functions, then $T_{\alpha}(f \circ g)(t)=t^{1-\alpha} g^{\prime}(t) f^{\prime}(g(t))$.

Moreover, some conformable fractional derivatives of certain functions can be found in [28]. The abovementioned properties will be utilized further in the forthcoming sections.

\subsection{Description of the functional variable method}

Consider the following general FPDE with four independent variables:

$P\left(u, \frac{\partial^{\alpha} u}{\partial t^{\alpha}}, \frac{\partial^{\alpha} u}{\partial x^{\alpha}}, \frac{\partial^{\alpha} u}{\partial y^{\alpha}}, \frac{\partial^{\alpha} u}{\partial z^{\alpha}}, \frac{\partial^{2 \alpha} u}{\partial t^{2 \alpha}}, \frac{\partial^{2 \alpha} u}{\partial x^{2 \alpha}}, \ldots\right)=0,0<\alpha \leq 1$

where $P$ is a polynomial of $u(x, y, z, t)$ and its fractional partial derivatives, in which the highest order derivatives and the nonlinear terms are involved.

The foremost steps of the FVM can be outlined as follows [18,19]:

Step 1: To find the exact solution of Eq. (2), we use the fractional complex transformation

$u(x, y, z, t)=u(\xi), \quad \xi=\frac{k_{1} x^{\alpha}}{\alpha}+\frac{k_{2} y^{\alpha}}{\alpha}+\frac{k_{3} z^{\alpha}}{\alpha}+\frac{c t^{\alpha}}{\alpha}$,

where $k_{1}, k_{2}, k_{3}$ and $c$ are nonzero arbitrary constants, to convert Eq. (2) into the following ordinary differential equation (ODE) of integer order:

$\tilde{P}\left(u, c u^{\prime}, k_{1} u^{\prime}, k_{2} u^{\prime}, k_{3} u^{\prime}, c^{2} u^{\prime \prime}, k_{1}{ }^{2} u^{\prime \prime}, \ldots\right)=0$, 
where $\tilde{P}$ is a polynomial in $u(\xi)$ and its total derivatives with respect to $\xi$.

Step 2: Let us make a transformation in which the unknown function $u(\xi)$ is considered as a functional variable in the form

$u_{\xi}=F(u)$

It is easy to find some higher order derivatives of $u(\xi)$ as follows:

$$
\begin{aligned}
& u_{\xi \xi}=F F^{\prime}=\frac{1}{2}\left(F^{2}\right)^{\prime}, \\
& u_{\xi \xi \xi}=\frac{1}{2}\left(F^{2}\right)^{\prime \prime} F=\frac{1}{2}\left(F^{2}\right)^{\prime \prime} \sqrt{F^{2}}, \\
& u_{\xi \xi \xi \xi}=\frac{1}{2}\left(\left(F^{2}\right)^{\prime \prime \prime} F^{2}+\frac{1}{2}\left(F^{2}\right)^{\prime \prime}\left(F^{2}\right)^{\prime}\right),
\end{aligned}
$$

and so on, where the prime denotes the derivative with respect to $u$.

Step 3: We substitute Eqs. (5) and (6) into Eq. (4) to reduce it to the following ODE:

$R\left(u, F, F^{\prime}, F^{\prime \prime}, \ldots\right)=0$.

Step 4: After integration, Eq. (7) provides the expression of $F$, and this in turn together with Eq. (5) gives the appropriate solutions to the original equation.

\section{APPLICATIONS}

In this section, we apply the functional variable method, which described in the previous section, to look for the exact solutions of two higher-dimensional space-time fractional equations of $\mathrm{KdV}$ type.

\subsection{The (3+1)-dimensional space-time fractional ZK equation}

Consider the (3+1)-dimensional space-time fractional ZK equation [24,25]

$D_{t}^{\alpha} u+a u D_{x}^{\alpha} u+D_{x}^{2 \alpha} u+D_{y}^{2 \alpha} u+D_{z}^{2 \alpha} u=0$,

where $0<\alpha \leq 1$ and $a$ is a nonzero constant.

To investigate Eq. (8) using the FVM, we use the fractional complex transformation given by Eq. (3) to reduce Eq. (8) into the following ODE:

$c u_{\xi}+a k_{1} u u_{\xi}+\left(k_{1}^{2}+k_{2}^{2}+k_{3}^{2}\right) u_{\xi \xi}=0$,

Integrating once w.r.t. $\xi$ and setting the constant of integration to zero, yields

$c u+\frac{a k_{1}}{2} u^{2}+\left(k_{1}^{2}+k_{2}^{2}+k_{3}^{2}\right) u_{\xi}=0$, 
Substituting Eq. (5) into Eq. (10), the function $F(u)$ reads

$F(u)=-\frac{c}{k_{1}^{2}+k_{2}^{2}+k_{3}^{2}} u\left(1+\frac{a k_{1}}{2 c} u\right)$

Separating the variables in Eq. (11) and then integrating, we obtain

$$
\int \frac{-d u}{u\left(1+\frac{a k_{1}}{2 c} u\right)}=\frac{c}{k_{1}^{2}+k_{2}^{2}+k_{3}^{2}}\left(\xi+\xi_{0}\right),
$$

where $\xi_{0}$ is a constant of integration. After completing the integration of Eq. (12), we get the following exact solutions:

$$
\begin{aligned}
& u_{1}(\xi)=-\frac{c}{a k_{1}}\left(1-\tanh \left(\frac{c}{2\left(k_{1}^{2}+k_{2}^{2}+k_{3}^{2}\right)}\left(\xi+\xi_{0}\right)\right),\right. \\
& u_{2}(\xi)=-\frac{c}{a k_{1}}\left(1-\operatorname{coth}\left(\frac{c}{2\left(k_{1}^{2}+k_{2}^{2}+k_{3}^{2}\right)}\left(\xi+\xi_{0}\right)\right) .\right.
\end{aligned}
$$

where $\xi=\frac{k_{1} x^{\alpha}}{\alpha}+\frac{k_{2} y^{\alpha}}{\alpha}+\frac{k_{3} z^{\alpha}}{\alpha}+\frac{c t^{\alpha}}{\alpha}$.

\subsection{The (2+1)-dimensional space-time fractional GZK-BBM equation}

Consider the (2+1)-dimensional space-time fractional GZK-BBM equation in the form [26]

$D_{t}^{\alpha} u+D_{x}^{\alpha} u+a D_{x}^{\alpha} u^{n}+b D_{x}^{\alpha}\left(D_{x}^{\alpha} D_{t}^{\alpha} u+D_{y}^{2 \alpha} u\right)=0, \quad n>1$

where $0<\alpha \leq 1$ and $a, b$ are nonzero constants.

To apply the FVM for Eq. (15). We exploit the fractional complex transformation

$u(x, y, t)=u(\xi), \quad \xi=\frac{k_{1} x^{\alpha}}{\alpha}+\frac{k_{2} y^{\alpha}}{\alpha}+\frac{c t^{\alpha}}{\alpha}$,

to convert Eq. (15) into the following ODE:

$\left(c+k_{1}\right) u_{\xi}+a k_{1}\left(u^{n}\right)_{\xi}+b k_{1}\left(k_{1} c u_{\xi \xi}+k_{2}^{2} u_{\xi \xi}\right)_{\xi}=0$,

Integrating once w.r.t. $\xi$ with zero constant of integration, we obtain

$\left(c+k_{1}\right) u+a k_{1} u^{n}+b k_{1}\left(k_{1} c+k_{2}^{2}\right) u_{\xi \xi}=0$,

Substituting Eqs. (6) into Eq. (18), yields 
$\left(F^{2}\right)^{\prime}=-\frac{2}{b k_{1}\left(k_{1} c+k_{2}^{2}\right)}\left[\left(c+k_{1}\right) u+a k_{1} u^{n}\right]$,

Integrating Eq. (19) w.r.t. $u$, we deduce the expression of the function $F(u)$ as follows

$$
F(u)=\sqrt{\frac{-\left(c+k_{1}\right)}{b k_{1}\left(k_{1} c+k_{2}^{2}\right)}} u \sqrt{1+\frac{2 a k_{1}}{\left(c+k_{1}\right)(n+1)} u^{n-1}},
$$

Separating the variables in Eq. (20) and then integrating, we obtain

$$
\int \frac{d u}{u \sqrt{1+\frac{2 a k_{1}}{\left(c+k_{1}\right)(n+1)} u^{n-1}}}=\sqrt{\frac{-\left(c+k_{1}\right)}{b k_{1}\left(k_{1} c+k_{2}^{2}\right)}}\left(\xi+\xi_{0}\right),
$$

where $\xi_{0}$ is a constant of integration. After completing the integration of Eq. (21), we can simply attain the following exact solutions:

(i) If $\frac{c+k_{1}}{b k_{1}\left(k_{1} c+k_{2}^{2}\right)}<0$, we have the following hyperbolic solutions:

$u_{1}(\xi)=\left\{-\frac{\left(c+k_{1}\right)(n+1)}{2 a k_{1}} \operatorname{sech}^{2}\left(\frac{n-1}{2} \sqrt{\frac{-\left(c+k_{1}\right)}{b k_{1}\left(k_{1} c+k_{2}^{2}\right)}}\left(\xi+\xi_{0}\right)\right)\right\}^{\frac{1}{n-1}}$,

$u_{2}(\xi)=\left\{\frac{\left(c+k_{1}\right)(n+1)}{2 a k_{1}} \operatorname{csch}^{2}\left(\frac{n-1}{2} \sqrt{\frac{-\left(c+k_{1}\right)}{b k_{1}\left(k_{1} c+k_{2}^{2}\right)}}\left(\xi+\xi_{0}\right)\right)\right\}^{\frac{1}{n-1}}$,

where $\xi=\frac{k_{1} x^{\alpha}}{\alpha}+\frac{k_{2} y^{\alpha}}{\alpha}+\frac{c t^{\alpha}}{\alpha}$.

(ii) If $\frac{c+k_{1}}{b k_{1}\left(k_{1} c+k_{2}^{2}\right)}>0$, we have the following trigonometric solutions:

$$
\begin{aligned}
& u_{3}(\xi)=\left\{-\frac{\left(c+k_{1}\right)(n+1)}{2 a k_{1}} \sec ^{2}\left(\frac{n-1}{2} \sqrt{\frac{\left(c+k_{1}\right)}{b k_{1}\left(k_{1} c+k_{2}^{2}\right)}}\left(\xi+\xi_{0}\right)\right)\right\}^{\frac{1}{n-1}}, \\
& u_{4}(\xi)=\left\{-\frac{\left(c+k_{1}\right)(n+1)}{2 a k_{1}} \csc ^{2}\left(\frac{n-1}{2} \sqrt{\frac{\left(c+k_{1}\right)}{b k_{1}\left(k_{1} c+k_{2}^{2}\right)}}\left(\xi+\xi_{0}\right)\right)\right\}^{\frac{1}{n-1}} .
\end{aligned}
$$


where $\xi=\frac{k_{1} x^{\alpha}}{\alpha}+\frac{k_{2} y^{\alpha}}{\alpha}+\frac{c t^{\alpha}}{\alpha}$.

\section{GRAPHICAL ILLUSTRATIONS}

In this section, with the aid of Maple software, we show the graphical representation of some results in Figs. 1-3 by assigning appropriate values to the unknown parameters in order to visualize the mechanism of Eqs. (8) and (15). Some physical interpretations are also presented.

\subsection{The (3+1)-dimensional space-time fractional ZK equation}

The profiles of the kink-shaped solution $u_{1}(\xi)$ given by Eq. (13) is shown in Fig. 1 when $y=0, z=1, a=1, \quad k_{1}=1.5, k_{2}=0.25, k_{3}=1, c=-2, \xi_{0}=0$ for various values of $\alpha$. We can observe that when the fractional derivative order $\alpha$ increased, the shape is closer to the known kink wave as the velocity of the propagation wave decreases. The kink wave keeps its height for various values of $\alpha$. It should also be pointed out that the solution $u_{2}(\xi)$ given by Eq. (14) is a singular kink solution.

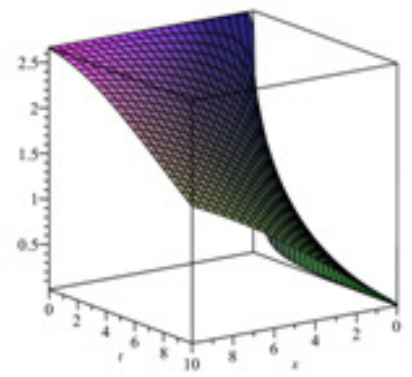

(a) $\alpha=0.25$

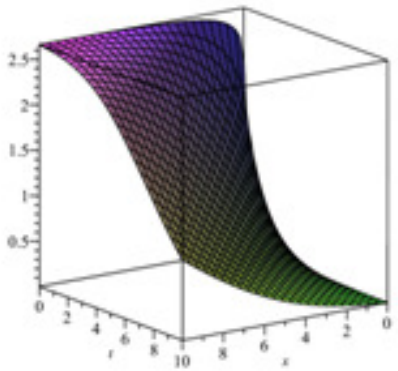

(b) $\alpha=0.5$

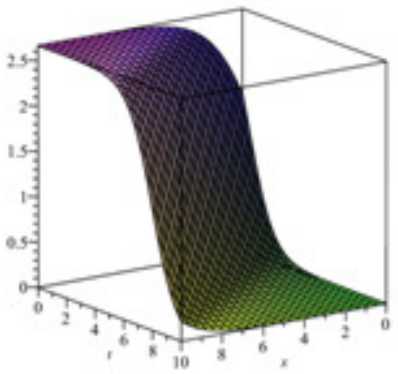

(c) $\alpha=1$

Figure 1. The kink solution corresponding to Eq. (13) for various values of $\alpha$

\subsection{The (2+1)-dimensional space-time fractional GZK-BBM equation}

The dynamics of the singular soliton solution $u_{2}(\xi)$ given by Eq. (23) is shown in Fig. 2 when $y=1, a=1, b=-2, k_{1}=1.25, k_{2}=-4, c=2, n=4, \xi_{0}=0$ for various values of $\alpha$. When $\alpha$ increased, the height of the wave changes as the velocity of the wave propagation decreases. Fig. 3 shows the motions of the periodic wave solution $u_{3}(\xi)$ given by Eq. (24) when $y=0, a=1$, $b=0.5, k_{1}=0.5, k_{2}=0.25, c=-2, n=4, \xi_{0}=0$. for various values of $\alpha$. When $\alpha$ increased, the height of the wave becomes lower as the velocity of the wave propagation decreases. It should also be mentioned that the solution $u_{1}(\xi)$ given by Eq. (22) is a bell-shaped soliton solution. 


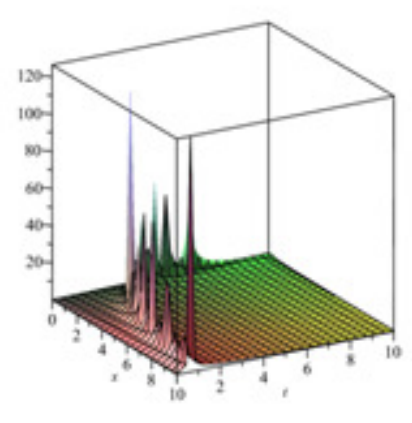

(a) $\alpha=0.25$

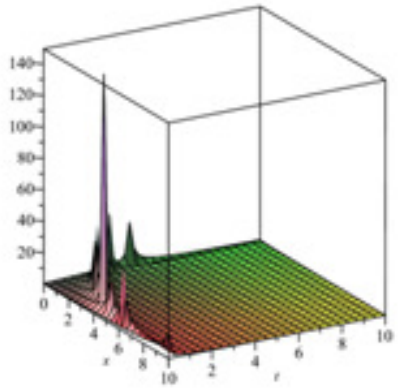

(b) $\alpha=0.5$

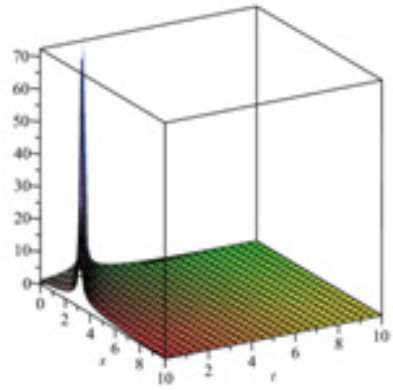

(c) $\alpha=1$

Figure 2. The singular soliton solution corresponding to Eq. (23) for various values of $\alpha$

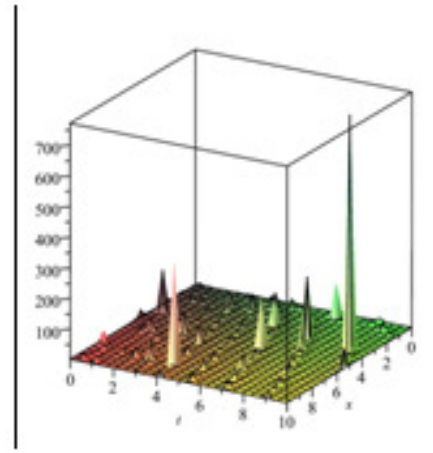

(a) $\alpha=0.25$

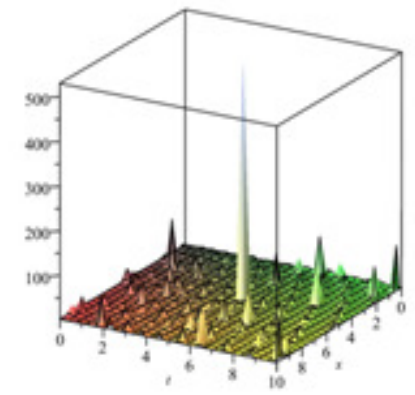

(b) $\alpha=0.5$

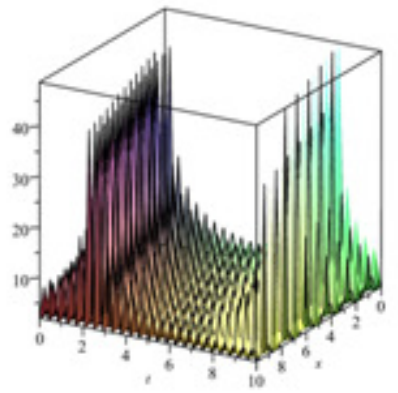

(c) $\alpha=1$

Figure 3. The periodic solution corresponding to Eq. (24) for various values of $\alpha$

\section{RESULTS AND DISCUSSION}

For the first time, the analytical solutions of the $(3+1)$-dimensional space-time fractional ZK equation and the (2+1)-dimensional space-time fractional GZK-BBM equation have been attained via the functional variable method, the fractional derivative has been described in the conformable sense. Consequently, we deduce that our solutions (13), (14), (22)-(25) are new and not discussed heretofore. It is remarkable that the obtained solutions in this article have potential physical meaning for the underlying equations. In addition to the physical meaning, these solutions can be used to measure the accuracy of numerical results and to help in the study of stability analysis.

\section{CONCLuSiOnS}

In this paper, we have successfully executed the functional variable method to attain new exact traveling wave solutions of a family of higher-dimensional space-time fractional KdV-type 
equations arising in mathematical physics, namely the (3+1)-dimensional space-time fractional ZK equation and the (2+1)-dimensional space-time fractional GZK-BBM equation. Two types of solutions including hyperbolic function solutions and trigonometric function solutions are obtained and numerically simulated in Figs. 1-3. The obtained solutions are significant to reveal the inner mechanism of the nonlinear physical phenomena that described by the aforementioned equations. It is shown that the FVM is straightforward, powerful and can be extended to handle many other higher-dimensional fractional partial differential equations as it maintains the reduced volume of computational work. With the aid of the Maple, we have verified our results.

\section{REFERENCES}

[1] Samko, S.G., Kilbas, A.A., Marichev, O.I., (1993) Fractional Integrals and Derivatives Theory and Applications. Gordonand Breach, New York.

[2] Podlubny, I., (1999) Fractional Differential Equations. Academic Press, San Diego.

[3] Zhang, S., Zong, Q.A., Liu, D., Gao, Q., (2010) A generalized exp-function method for fractional Riccati differential equations. Commun. Fract. Calc. 1, 48-51.

[4] Bekir, A., Guner, O., Cevikel, A.C., (2013) Fractional complex transform and exp-function methods for fractional differential equations. Abstr. Appl. Anal. 2013, 426462.

[5] El-Sayed, A.M.A., Rida, S.Z., Arafa, A.A.M., (2009) Exact solutions of fractional-order biological population model. Commun. Theor. Phys. 52, 992 -996.

[6] Shang, N., Zheng, B., (2013) Exact solutions for three fractional partial differential equations by the method. Int. J. Appl. Math. 43 (3), 114-119.

[7] Ekici, M., Mirzazadeh, M., Eslami, M., Zhou, Q., Moshokoa, S.P., Biswas, A., Belic, M., (2016) Optical soliton perturbation with fractional-temporal evolution by first integral method with conformable fractional derivatives. Optik 127, 10659-10669.

[8] Eslami, M., Rezazadeh, H., (2016) The first integral method for Wu-Zhang system with conformable time-fractional derivative. Calcolo 53, 475-485.

[9] Inc, M., (2008) The approximate and exact solutions of the space- and time-fractional Burgers equations with initial conditions by variational iteration method. J. Math. Anal. Appl. 345, 476-484.

[10] Aminikhah, H., Sheikhani, A.H.R., Rezazadeh, H., (2016) Sub-equation method for the fractional regularized long-wave equations with conformable fractional derivatives. Scientia Iranica B 23 (3), 1048-1054.

[11] Zheng, B., Wen, C., (2013) Exact solutions for fractional partial differential equations by a new fractional sub-equation method. Adv. Difference Equ. 2013, 199.

[12] Kaplan, M., Bekir, A., Akbulut, A., Aksoy, E., (2015) The modified simple equation method for nonlinear fractional differential equations. Rom. Jour. Phys. 60, 1374-1383.

[13] Tasbozan, O., Çenesiz, Y., Kurt, A., (2016) New solutions for conformable fractional Boussinesq and combined KdV-mKdV equations using Jacobi elliptic function expansion method. Eur. Phys. J. Plus $131,244$. 
[14] Demiray, S.T., Pandir, Y., Bulut, H., (2014) The investigation of exact solutions of nonlinear timefractional Klein-Gordon equation by using generalized Kudryashov method. AIP Conf. Proc. 1637, $283-289$.

[15] Demiray, S.T., Pandir, Y., Bulut, H., (2014) Generalized Kudryashov method for time-fractional differential equations. Abstr. Appl. Anal. 2014, 901540.

[16] Zerarka, A., Ouamane, S., Attaf, A., (2010) On the functional variable method for finding exact solutions to a class of wave equations. App. Math. and Com. 217, 2897-2904.

[17] Zerarka, A., Ouamane, S., (2010) Application of the functional variable method to a class of nonlinear wave equations. World J. Model. Simul. 6 (2), 150-160.

[18] Liu, W., Chen, K., (2013) The functional variable method for finding exact solutions of some nonlinear time-fractional differential equations. Pramana J. Phys., 81 (3), 377-384.

[19] Bekir, A., Güner, Ö., Aksoy, E., Pandir, Y., (2015) Functional variable method for the nonlinear fractional differential equations. AIP Conf. Proc. 1648, 730001.

[20] Khan, K., Akbar, M.A., (2015) Study of functional variable method for finding exact solutions of nonlinear evolution equations. Walailak J. Sci \& Tech. 12 (11), 1031-1042.

[21] Mirzazadeh, M., Eslami, M., (2013) Exact solutions for nonlinear variants of Kadomtsev-Petviashvili $(\mathrm{n}, \mathrm{n})$ equation using functional variable method. Pramana J. Phys. 81, 911-24.

[22] Bekir, A., San, S., (2013) Periodic, hyperbolic and rational function solutions of nonlinear wave equations. Appl. Math. Inf. Sci. Lett. 1 (3), 97-101.

[23] Wazwaz, A.M., (2009) Partial Differential Equations and Solitary Waves Theory (Higher Education Press, Beijing) P. 503.

[24] Zakharov, V.E., Kuznetsov, E.A., (1974) Three-dimensional solitons. Sov. Phys. 39, 285-286.

[25] Das, G.C., Sarma, J., Gao, Y.T., Uberoi, C., (2000) Dynamical behavior of the soliton formation and propagation in magnetized plasma. Phys. Plasmas 7, 2374- 2380.

[26] Wazwaz, A.M., (2005) Compact and noncompact physical structures for the ZK-BBM equation. App. Math. Comput. 169, 713-725.

[27] Oliveira, E.C. \& Machado, J.A.T., (2014) A review of definitions of fractional derivatives and Integral, Math. Probl. Eng. 2014238459.

[28] Khalil, R., Horani, AL.M., Yousef, A., Sababheh, M., (2014) A new definition of fractional derivative. J. Comput. Appl. Math. 264, 65-70.

[29] Abu Hammad, M., Khalil, R., (2014) Conformable fractional heat differential equation. Int. J. Pure Appl. Math. 94 (2), 215-221.

[30] Abdeljawad, T., (2015) On conformable fractional calculus. J. Comput. Appl. Math. 279 (1), 57-66. 


\section{AUTHOR}

Mohammed O. Al-Amr was born on January 29th, 1986 in Mosul, Iraq. He received his B.Sc. in Mathematics from University of Mosul in 2007. He received his M.Sc. in Mathematics from University of Mosul in 2013 and studied in the field of "Numerical Analysis". Since 2013, he has been an assistant lecturer at University of Mosul. He published many papers in reputable scientific journals. He serves as a reviewer and editorial member of many scientific journals. He is a member of many international scientific associations. His main research interests are numerical analysis, partial differential equations, semi-analytical methods, stability analysis, traveling wave analysis, theory of solitons.

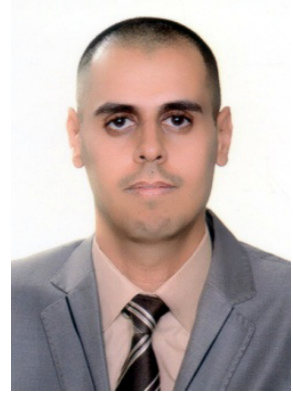

